\title{
BDDC for Nonsymmetric Positive Definite and Symmetric Indefinite Problems*
}

\author{
Xuemin $\mathrm{Tu}^{1}$ and Jing $\mathrm{Li}^{2}$ \\ 1 Department of Mathematics, University of California and Lawrence Berkeley \\ National Laboratory, Berkeley, CA 94720-3840 xuemin@math.berkeley.edu \\ 2 Department of Mathematical Sciences, Kent State University, Kent, OH 44242 \\ li@emath.kent.edu
}

Summary. The balancing domain decomposition methods by constraints are extended to solving both nonsymmetric, positive definite and symmetric, indefinite linear systems. In both cases, certain nonstandard primal constraints are included in the coarse problems of BDDC algorithms to accelerate the convergence. Under the assumption that the subdomain size is small enough, a convergence rate estimate for the GMRES iteration is established that the rate is independent of the number of subdomains and depends only slightly on the subdomain problem size. Numerical experiments for several two-dimensional examples illustrate the fast convergence of the proposed algorithms.

\section{Introduction}

Domain decomposition methods have been widely used and studied for solving large sparse linear systems arising from finite element discretization of partial differential equations, see [16] and the references therein. The balancing domain decomposition methods by constraints (BDDC) were introduced by Dohrmann [6], see also [9] and [5] for related algorithms. These algorithms originally were designed for the symmetric, positive definite systems. The BDDC methods have also been extended to solving saddle point problems, e.g., Stokes equations [12], nearly incompressible elasticity [7], and the flow in porous media $[17,18]$.

Cai and Widlund $[2,3,4]$ studied overlapping Schwarz methods for nonsymmetric and indefinite problems, using a perturbation approach in their analysis, and established that the convergence rates of the two-level overlapping Schwarz methods are independent of the mesh size if the coarse mesh is fine enough.

In this paper, we extend BDDC algorithms to nonsymmetric, positive definite linear systems arising from finite element discretization of advectiondiffusion equations, and to symmetric, indefinite systems arising from finite

*This work was supported by the U.S. Department of Energy under Contract No. DE-AC02-05CH11231. 
element discretization of Helmholtz equations. A preconditioned GMRES iteration is used. In the preconditioning step of each iteration, a partially subassembled finite element problem is solved, for which only the coarse level, primal interface degrees of freedom are shared by neighboring subdomains. A perturbation approach is used in our analysis to handle the non-symmetry or indefiniteness of the problems. A key point in the analysis is the error bound for a partially sub-assembled finite element problem; we view this partially sub-assembled finite element problem as a non-conforming finite element approximation.

\section{Finite element discretization}

Let $\widehat{W} \subset H_{0}^{1}(\Omega)$ be the standard continuous, piecewise linear finite element function space on a shape-regular triangulation of $\Omega$. In this paper, we use the same notation, e.g., $u$, to denote both a finite element function and the vector of its coefficients with respect to the finite element basis; we will also use the same notation to denote the space of finite element functions and the space of their corresponding vectors, e.g., $\widehat{W}$. In this paper, $C$ always represents a generic positive constant independent of all the parameters and mesh size.

\subsection{Nonsymmetric, positive definite problems}

We consider the following second order scalar advection-diffusion problem in a bounded polyhedral domain $\Omega \in \mathbf{R}^{d}, \mathrm{~d}=2,3$,

$$
\left\{\begin{aligned}
L u:=-\nu \triangle u+\mathbf{a} \cdot \nabla u+c u & =f, & & \text { in } \Omega, \\
u & =0, & & \text { on } \partial \Omega .
\end{aligned}\right.
$$

Here the viscosity $\nu$ is a positive constant. The velocity field $\mathbf{a}(x) \in\left(L^{\infty}(\Omega)\right)^{d}$ and $\nabla \cdot \mathbf{a}(x) \in L^{\infty}(\Omega)$. The reaction coefficient $c(x) \in L^{\infty}(\Omega)$ and $f(x) \in$ $L^{2}(\Omega)$. We define $\tilde{c}(x)=c(x)-\frac{1}{2} \nabla \cdot \mathbf{a}(x)$ and assume that there exists a positive constant $c_{0}$ such that

$$
\tilde{c}(x) \geq c_{0}>0, \quad \forall x \in \Omega .
$$

We focus on studying the dependence on $\nu$ of the performance of our algorithms and assume that all other parameters in the operator $L$ are of order $O(1)$.

The bilinear form associated with the operator $L$ is defined, for functions in the space $H_{0}^{1}(\Omega)$, by $a_{o}(u, v)=\int_{\Omega}(\nu \nabla u \cdot \nabla v+\mathbf{a} \cdot \nabla u v+c u v) d x$, which is positive definite under assumption (2). The weak solution $u \in H_{0}^{1}(\Omega)$ of (1) satisfies

$$
a_{o}(u, v)=\int_{\Omega} f v d x, \quad \forall v \in H_{0}^{1}(\Omega) .
$$


We assume that the weak solution $u$ of the original problem (1), as well as the weak solution of the adjoint problem $L^{*} u=-\nu \Delta u-\nabla \cdot(\mathbf{a} u)+c u=f$, satisfies the regularity result,

$$
\|u\|_{H^{2}(\Omega)} \leq \frac{C}{\nu}\|f\|_{L^{2}(\Omega)},
$$

where $C$ is a positive constant independent of $\nu$. Here we assume that $\|u\|_{H^{2}(\Omega)}$ grows proportionally with a decrease of the viscosity $\nu$.

It is well known that the original bilinear form $a_{o}(\cdot, \cdot)$ has to be stabilized to remove spurious oscillations in the finite element solution for advectiondominated problems. Here, we follow $[10,15]$ and consider the Galerkin/leastsquares method (GALS).

The stabilized finite element problem for solving (3) is: find $u \in \widehat{W}$, such that for all $v \in \widehat{W}$,

$$
a(u, v):=a_{o}(u, v)+\int_{\Omega} C(x) L u L v d x=\int_{\Omega} f v d x+\int_{\Omega} C(x) f L v d x,
$$

where $C(x)$ is a positive function which depends on the local element Peclet number; see [19] for details. We note that for all piecewise linear finite element functions $u, L u=-\nu \triangle u+\mathbf{a} \cdot \nabla u+c u=\mathbf{a} \cdot \nabla u+c u$, on each element. We define $C_{s}=\max _{x \in \Omega}|C(x)|$ and $C_{m}=\min _{x \in \Omega}|C(x)|$.

The system of linear equations corresponding to the stabilized finite element problem (5) is denoted by

$$
A u=f,
$$

where the coefficient matrix $A$ is nonsymmetric but positive definite.

\subsection{Symmetric, indefinite problems}

We consider the solution of the following partial differential equation on a bounded polyhedral domain $\Omega \in \mathbf{R}^{d}, \mathrm{~d}=2,3$,

$$
\left\{\begin{aligned}
-\Delta u-\sigma^{2} u=f, & \text { in } \Omega, \\
u=0, & \text { on } \partial \Omega,
\end{aligned}\right.
$$

where $\sigma$ is a real constant. The weak solution $u \in H_{0}^{1}(\Omega)$ of $(7)$ satisfies

$$
a(u, v)=(f, v), \quad \forall v \in H_{0}^{1}(\Omega),
$$

where $a(u, v)=\int_{\Omega} \nabla u \cdot \nabla v-\sigma^{2} u v$, and $(f, v)=\int_{\Omega} f v$. Under the assumption that (8) has a unique solution, we can prove the following regularity result for the weak solution, cf. [11],

$$
\|u\|_{H^{1+\gamma}(\Omega)} \leq C\left(1+\frac{\sigma^{2}}{\left|\lambda_{*}-\sigma^{2}\right|}\right)\|f\|_{L_{2}(\Omega)}
$$


where $\lambda_{*}$ is the eigenvalue of the corresponding Laplace operator, closest to $\sigma^{2}$. The results hold for $\gamma=1$, if $\Omega$ is convex. In this paper we assume that $\sigma^{2}$ is bounded away from the eigenvalues of the Laplace operator such that the problem is well posed. Therefore we have $\|u\|_{H^{1+\gamma}(\Omega)} \leq C\left(1+\sigma^{2}\right)\|f\|_{L_{2}(\Omega)}$.

The finite element solution for solving (8) is: find $u \in \widehat{W}$, such that

$$
a(u, v)=(f, v), \quad \forall v \in \widehat{W} .
$$

The resulting system of linear equations has the form

$$
A u=\left(K-\sigma^{2} M\right) u=f,
$$

where $K$ is the stiffness matrix, and $M$ is the mass matrix.

\section{The BDDC preconditioners}

We decompose the space $\widehat{W}$ into $W_{I} \oplus \widehat{W}_{\Gamma}$, where $W_{I}$ is the product of local subdomain spaces $W_{I}^{(i)}, i=1,2, \ldots, N$, corresponding to the subdomain interior variables. $\widehat{W}_{\Gamma}$ is the subspace corresponding to the variables on the subdomain interface $\Gamma$. The original discrete problem (6) can be written as: find $u_{I} \in W_{I}$ and $u_{\Gamma} \in \widehat{W}_{\Gamma}$, such that

$$
\left[\begin{array}{ll}
A_{I I} & A_{I \Gamma} \\
A_{\Gamma I} & A_{\Gamma \Gamma}
\end{array}\right]\left[\begin{array}{l}
u_{I} \\
u_{\Gamma}
\end{array}\right]=\left[\begin{array}{l}
f_{I} \\
f_{\Gamma}
\end{array}\right]
$$

where $A_{I I}$ is block diagonal with one block for each subdomain, and $A_{\Gamma \Gamma}$ corresponds to the subdomain interface variables and is assembled from subdomain matrices across the subdomain interfaces.

Eliminating the subdomain interior variables $u_{I}$ from (12), we have the Schur complement problem

$$
S_{\Gamma} u_{\Gamma}=g_{\Gamma}
$$

where $S_{\Gamma}=A_{\Gamma \Gamma}-A_{\Gamma I} A_{I I}^{-1} A_{I \Gamma}$, and $g_{\Gamma}=f_{\Gamma}-A_{\Gamma I} A_{I I}^{-1} f_{I}$.

A partially sub-assembled finite element space $\widetilde{W}$ is defined by $\widetilde{W}=W_{I} \oplus$ $\widetilde{W}_{\Gamma}$. Here $\widetilde{W}_{\Gamma}$ contains the coarse level, continuous primal interface degrees of freedom, in the subspace $\widehat{W}_{\Pi}$, which are shared by neighboring subdomains, and the remaining dual subdomain interface degrees of freedom which are in general discontinuous across the subdomain interfaces. Then a partially sub-assembled problem matrix $\widetilde{A}$ is defined by

$$
\left[\begin{array}{ll}
A_{I I} & \widetilde{A}_{I \Gamma} \\
\widetilde{A}_{\Gamma I} & \widetilde{A}_{\Gamma \Gamma}
\end{array}\right],
$$

where $\widetilde{A}_{\Gamma \Gamma}$ is assembled only with respect to the coarse level primal degrees of freedom across the interface. 
Correspondingly, a partially sub-assembled Schur complement $\widetilde{S}_{\Gamma}$ is defined by $\widetilde{S}_{\Gamma}=\widetilde{A}_{\Gamma \Gamma}-\widetilde{A}_{\Gamma I} A_{I I}^{-1} \widetilde{A}_{I \Gamma}$. From the definition of $S_{\Gamma}$ and $\widetilde{S}_{\Gamma}$, we see that $S_{\Gamma}$ can be obtained from $\widetilde{S}_{\Gamma}$ by assembling with respect to the dual interface variables, i.e.,

$$
S_{\Gamma}=\widetilde{R}_{\Gamma}^{T} \widetilde{S}_{\Gamma} \widetilde{R}_{\Gamma}
$$

where $\widetilde{R}_{\Gamma}$ is the injection operator from the space $\widehat{W}_{\Gamma}$ into $\widetilde{W}_{\Gamma}$. We also define $\widetilde{R}_{D, \Gamma}=D \widetilde{R}_{\Gamma}$, where $D$ is a diagonal scaling matrix. The diagonal elements of $D$ equal 1 , for the rows of the primal interface variables, and equal $\delta_{i}^{\dagger}(x)$ for the others. Here, for a subdomain interface node $x$, the inverse counting function $\delta_{i}^{\dagger}(x)$ is defined by $\delta_{i}^{\dagger}(x)=1 / \operatorname{card}\left(\mathcal{N}_{x}\right)$, where $\mathcal{N}_{x}$ is the set of indices of the subdomains which have $x$ on their boundaries and $\operatorname{card}\left(\mathcal{N}_{x}\right)$ is the number of the subdomains in the set $\mathcal{N}_{x}$.

The preconditioned interface problem in our BDDC algorithm is

$$
\widetilde{R}_{D, \Gamma}^{T} \widetilde{S}_{\Gamma}^{-1} \widetilde{R}_{D, \Gamma} S_{\Gamma} u_{\Gamma}=\widetilde{R}_{D, \Gamma}^{T} \widetilde{S}_{\Gamma}^{-1} \widetilde{R}_{D, \Gamma} g_{\Gamma} .
$$

A GMRES iteration is used to solve (14). In each iteration, to multiply $S_{\Gamma}$ by a vector, subdomain Dirichlet boundary problems need to be solved; to multiply $\widetilde{S}_{\Gamma}^{-1}$ by a vector, a partially sub-assembled finite element problem with the coefficient matrix $\widetilde{A}$ needs to be solved, which requires solving subdomain Neumann/Robin boundary problems and a coarse level problem; cf. $[13,19]$. After obtaining the interface solution $u_{\Gamma}$, we find $u_{I}$ by solving subdomain Dirichlet problems.

Another alternative of the BDDC algorithm is to iterate on the full set of variables, instead of on the subdomain interface variables. This alternative preconditioned BDDC operator is of the form

$$
\left(\widetilde{R}_{D}^{T}-\mathcal{H} J_{D}\right) \widetilde{A}^{-1}\left(\widetilde{R}_{D}-J_{D}^{T} \mathcal{H}^{T}\right) A,
$$

where $\widetilde{R}_{D}$ is a scaled injection operator from $\widehat{W}$ onto $\widetilde{W}$ with the scaling on the subdomain interface defined in the same way as for $\widetilde{R}_{D, \Gamma}$ discussed above. $J_{D}$ is a map from $\widetilde{W}$ to itself. For any $w \in \widetilde{W}$, the component of $J_{D} w$, for the subdomain $\Omega_{i}$, is defined by

$$
\left(J_{D} w(x)\right)^{(i)}=\sum_{j \in \mathcal{N}_{x}} \delta_{j}^{\dagger}(x)\left(w^{(i)}(x)-w^{(j)}(x)\right), \quad \forall x \in \Gamma \cap \partial \Omega_{i},
$$

where $J_{D} w$ vanishes in the interior of the subdomain and for the coarse level component. The component of $J_{D}^{T} w$ for subdomain $\Omega_{i}$ is then given by

$$
\left(J_{D}^{T} w(x)\right)^{(i)}=\sum_{j \in \mathcal{N}_{x}}\left(\delta_{j}^{\dagger}(x) w^{(i)}(x)-\delta_{i}^{\dagger}(x) w^{(j)}(x)\right), \quad \forall x \in \Gamma \cap \partial \Omega_{i} .
$$

The subdomain interior and the coarse level primal components of $J_{D}^{T} w$ also vanish. The operator $\mathcal{H}$ in (15) is direct sum of the subdomain discrete harmonic extensions $\mathcal{H}^{(i)}$, where $\mathcal{H}^{(i)}=-K_{I I}^{(i)-1} K_{I \Gamma}^{(i)}, i=1,2, \ldots, N$. H $J_{D}$ represents the discrete harmonic extension of the jump of the dual interface variables to the interior of the subdomains. 


\section{Convergence rate analysis}

The GMRES iteration is used in our BDDC algorithm to solve the preconditioned system of linear equations. To estimate the convergence rate of the GMRES iteration, we use the following result, cf. [8],

Theorem 1. Let $c_{1}$ and $C_{2}$ be two positive parameters, $\langle\cdot, \cdot\rangle_{\Lambda}$ be an inner product defined in a vector space $V, T$ be a linear operator defined on $V$. If for all $v \in V$,

$$
\begin{aligned}
c_{1}\langle v, v\rangle_{\Lambda} & \leq\langle v, T v\rangle_{\Lambda}, \\
\langle T v, T v\rangle_{\Lambda} & \leq C_{2}\langle v, v\rangle_{\Lambda},
\end{aligned}
$$

then

$$
\frac{\left\|r_{m}\right\|_{\Lambda}}{\left\|r_{0}\right\|_{\Lambda}} \leq\left(1-\frac{c_{1}^{2}}{C_{2}}\right)^{m / 2}
$$

where $r_{m}$ is the residual at step $m$ of the GMRES iteration applied to T.

Remark 1. The convergence rate of the GMRES iteration using the standard $L_{2}$ inner product will not be estimated in this paper. In our numerical experiments, we have found that using the $\Lambda$ inner product or the standard $L_{2}$ inner product gives the same convergence rate. For a study of the convergence rates of the GMRES iteration for an additive Schwarz method in the Euclidean and energy norms, see Sarkis and Szyld [14].

In the following analysis, we focus on obtaining the two positive constants $c_{1}$ and $C_{2}$ defined in (16) and (17), with respect to appropriate norms.

\subsection{Nonsymmetric, positive cases}

The preconditioned BDDC operator for solving the nonsymmetric, positive definite problem (6) is $T=\widetilde{R}_{D, \Gamma}^{T} \widetilde{S}_{\Gamma}^{-1} \widetilde{R}_{D, \Gamma} S_{\Gamma}$, defined on the subdomain interface variable space $\widehat{W}_{\Gamma}$. The inner product in the GMRES iteration is defined by $\Lambda=S_{\Gamma}$. We assume

Assumption 2 For two-dimensional problems, the coarse level primal subspace $\widehat{W}_{\Pi}$ contains all subdomain corner degrees of freedom, and for each edge $\mathcal{E}^{k}$, one edge average degree of freedom and two edge flux average degrees of freedom such that for any $w \in W$,

$$
\int_{\mathcal{E}^{k}} w^{(i)} d s, \quad \int_{\mathcal{E}^{k}} \mathbf{a} \cdot \mathbf{n} w^{(i)} d s, \quad \text { and } \int_{\mathcal{E}^{k}} \mathbf{a} \cdot \mathbf{n} w^{(i)} s d s,
$$

respectively, are the same (with a difference of factor -1 corresponding to opposite normal directions) for the two subdomains $\Omega_{i}$ that share $\mathcal{E}^{k}$. 
For three dimensional problems, $\widehat{W}_{\Pi}$ contains all subdomain corner degrees of freedom, and for each face $\mathcal{F}^{l}$, one face average degree of freedom and two face flux average degrees of freedom, and for each edge $\mathcal{E}^{k}$, one edge average degree of freedom, such that for any $w \in \widetilde{W}$,

$\int_{\mathcal{F}^{l}} I_{h}\left(\vartheta_{\mathcal{F}^{l}} w^{(i)}\right) d s, \quad \int_{\mathcal{F}^{l}} \mathbf{a} \cdot \mathbf{n} I_{h}\left(\vartheta_{\mathcal{F}^{l}} w^{(i)}\right) d s$, and $\int_{\mathcal{F}^{l}} \mathbf{a} \cdot \mathbf{n} I_{h}\left(\vartheta_{\mathcal{F}^{l}} w^{(i)}\right) s d s$, respectively, are the same (with a difference of factor -1 corresponding to opposite normal directions) for the two subdomains $\Omega_{i}$ that share the face $\mathcal{F}^{l}$, and

$$
\int_{\mathcal{E}^{k}} I_{h}\left(\vartheta_{\mathcal{E}^{k}} w^{(i)}\right) d s
$$

are the same for all subdomains $\Omega_{i}$ that share the edge $\mathcal{E}^{k}$. Here $\vartheta_{\mathcal{F} l}$ and $\vartheta_{\mathcal{E}^{k}}$ are the standard finite element face and edge cutoff functions, respectively.

Theorem 3. Let Assumption 2 hold. If $h$ is sufficiently small, there then exist positive constants $C_{1}, C_{2}$, and $C_{3}$, which are independent of $H, h$, and $\nu$, such that for all $u_{\Gamma} \in \widehat{W}_{\Gamma}$,

$$
\left\langle T u_{\Gamma}, T u_{\Gamma}\right\rangle_{\Lambda} \leq C_{1} \frac{\Phi^{4}(H, h)}{\nu^{2} \max \left(\nu, C_{m}\right)}\left\langle u_{\Gamma}, u_{\Gamma}\right\rangle_{\Lambda},
$$

and

$$
c_{0}\left\langle u_{\Gamma}, u_{\Gamma}\right\rangle_{\Lambda} \leq \frac{C_{2}}{\max \left(\nu, C_{m}\right)}\left\langle u_{\Gamma}, T u_{\Gamma}\right\rangle_{\Lambda},
$$

where $\Phi(H, h)=C(1+\log (H / h))$. For two dimensions

$$
c_{0}=1-C_{3} \frac{\max \left(\sqrt{\nu}, \sqrt{C_{s}}\right) \max \left(H \nu, H^{2}\right)}{\nu^{3} \max \left(\nu^{2} \sqrt{\nu}, C_{m}^{2} \sqrt{C_{m}}\right)} \frac{H}{h} \Phi^{2}(H, h),
$$

and for three dimensions

$$
c_{0}=1-C_{3} \frac{\max \left(\sqrt{\nu}, \sqrt{C_{s}}\right) \max \left(H \nu, H^{2}, \sqrt{H h}\right)}{\nu^{3} \max \left(\nu^{2} \sqrt{\nu}, C_{m}^{2} \sqrt{C_{m}}\right)} \frac{H}{h} \Phi^{2}(H, h)(1+\log (H / h)) .
$$

\subsection{Symmetric, indefinite cases}

The preconditioned BDDC operator for solving the symmetric, indefinite problem (11) is $T=\left(\widetilde{R}_{D}^{T}-\mathcal{H} J_{D}\right) \widetilde{A}^{-1}\left(\widetilde{R}_{D}-J_{D}^{T} \mathcal{H}^{T}\right) A$, defined on $\widehat{W}$. The inner product in the GMRES iteration is defined by $\Lambda=K+\sigma^{2} M$. We assume

Assumption 4 The coarse level primal subspace $\widehat{W}_{\Pi}$ contains all subdomain corner degrees of freedom, one edge average degree of freedom on each edge corresponding to restriction of the plane wave $\cos (\sigma \theta \cdot x)$ on the edge with the unit direction vector $\theta$ chosen orthogonal to the edge, and, for three dimensional problems, one face average degree of freedom on each subdomain boundary face corresponding to restriction of the plane wave $\cos (\sigma \theta \cdot x)$ on the face with $\theta$ chosen orthogonal to the face. 
Theorem 5. Let Assumptions 4 hold. If $\sigma\left(1+\sigma^{2}\right)(1+\Phi(H, h)) H^{\gamma} C_{L}(H, h)$ is sufficiently small, then for all $u \in \widehat{W}$,

$$
\begin{aligned}
c\langle u, u\rangle_{\Lambda} & \leq\langle u, T u\rangle_{\Lambda}, \\
\langle T u, T u\rangle_{\Lambda} & \leq C\left(1+\sigma^{2} H^{2}\right)\left(1+\Phi(H, h)^{2}\right)\langle u, u\rangle_{\Lambda},
\end{aligned}
$$

where $c$ and $C$ are positive constants independent of $\sigma, H$, and $h . \Phi(H, h)$ is defined in Theorem 3. $C_{L}(H, h)$ equals $(1+\log (H / h))$ for three-dimensional problems, and equals 1 for two-dimensional problems.

\section{Numerical experiments}

\subsection{Nonsymmetric, positive definite cases}

We test our BDDC algorithm by solving the advection-diffusion equation (1) on the square domain $\Omega=[-1,1]^{2}$. The domain $\Omega$ is decomposed into square subdomains and each subdomain into uniform triangles. Piecewise linear finite elements are used in our experiments. We take $f=0$ and $c=10^{-4}$ in (1) in our example. We choose the most difficult one from the three examples, which were used by Toselli [15] for testing his FETI algorithms. Here the velocity field is $\mathbf{a}=(y,-x)$. The boundary condition is given by:

$$
u=1, \text { for }\left\{\begin{array}{l}
y=-1 \quad 0<x \leq 1, \\
y=1, \quad 0<x \leq 1, \\
x=1, \quad-1 \leq y \leq 1,
\end{array} \quad \text { with } \quad u=0, \quad \text { elsewhere on } \partial \Omega .\right.
$$

In the GMRES iteration, the $L_{2}$ inner product is used and the iteration is stopped when the residual is reduced by $10^{-6}$.

In our experiments, we test three different choices of the coarse spaces in the algorithms. In our first test, we test the Robin-Robin algorithms, which is closely related to our BDDC algorithms, see [1]. In our second test, the coarse level primal variables of our BDDC algorithms are only those at the subdomain corners and the subdomain edge averages; no additional continuity constraints corresponding to the flux are enforced across the subdomain edges. This choice of the coarse level primal space does not satisfy Assumption 2. In our last test, in addition to the primal constraints used in the second test, we also include in the coarse level problem two weighted edge average degrees of freedom corresponding to flux continuity constraints for each subdomain edge, as required in Assumption 2. In the following tables, we represent these three different algorithms by RR, BDDC-1, and BDDC-2, respectively.

Table 1 gives the iteration counts of the three algorithms for different number of subdomains with a fixed subdomain problem size. We see that BDDC-2 converges much faster than BDDC-1 and the Robin-Robin algorithm. For the cases where $\nu>10^{-5}$, the iteration counts are almost independent 
Table 1. Iteration counts for nonsymmetric, positive definite problems with $H / h=$ 6 and changing number of subdomains.

\begin{tabular}{|ccccc|}
\hline \multirow{2}{*}{$\nu$} & \multirow{2}{*}{ \# subdomains } & $\mathrm{RR}$ & BDDC-1 & BDDC-2 \\
\cline { 3 - 5 } $1 e-2$ & $8 \times 8$ & 22 & 9 & 3 \\
& $16 \times 16$ & 31 & 7 & 3 \\
& $32 \times 32$ & 49 & 6 & 3 \\
\hline \multirow{3}{*}{$1 e-4$} & $8 \times 8$ & 114 & 67 & 12 \\
& $16 \times 16$ & 251 & 111 & 14 \\
& $32 \times 32$ & 475 & 112 & 14 \\
$1 e-6$ & $8 \times 8$ & 145 & 86 & 14 \\
& $16 \times 16$ & 389 & 199 & 18 \\
& $32 \times 32$ & $>500$ & 434 & 26 \\
\hline
\end{tabular}

Table 2. Iteration counts for nonsymmetric, positive definite problems with $4 \times 4$ subdomains and changing $H / h$.

\begin{tabular}{|ccccc|}
\hline \multirow{2}{*}{$\nu$} & $H / h$ & \multicolumn{3}{c|}{ Iteration Count } \\
\cline { 2 - 5 } $1 e-2$ & 12 & 17 & BDDC-1 & BDDC-2 \\
& 24 & 18 & 12 & 4 \\
& 48 & 20 & 13 & 4 \\
\hline \multirow{3}{*}{$1 e-4$} & 12 & 83 & 72 & 26 \\
& 24 & 110 & 104 & 39 \\
& 48 & 128 & 122 & 45 \\
\hline \multirow{3}{*}{$1 e-6$} & 12 & 100 & 87 & 34 \\
& 24 & 180 & 165 & 88 \\
& 48 & 296 & 290 & 142 \\
\hline
\end{tabular}

of the number of subdomains. Even when the viscosity $\nu$ goes to zero, the convergence of BDDC-2 is still very fast, while the convergence rates of BDDC1 and the Robin-Robin algorithm are not satisfactory at all.

From Table 2, we see that the iteration counts of all the algorithms increase with an increase of the subdomain problem size; the increase for BDDC-2 is the smallest.

\subsection{Symmetric and indefinite cases}

The problem ( 7 ) is solved on a $2 \pi$ by $2 \pi$ square domain with Dirichlet boundary conditions $u=1$ on the four sides of the square and with $f=0$. Q1 finite elements are used and the original square domain is decomposed uniformly 
into square subdomains. In the GMRES iteration, the $\langle\cdot, \cdot\rangle_{K+\sigma^{2} M}$ inner product is used; using $L_{2}$ inner product gives the same convergence rates. The iteration is stopped when the residual is reduced by $10^{-6}$.

In our experiments, we test three different choices of the coarse level primal space in our BDDC algorithm. In our first test, the coarse level primal variables are only those at the subdomain corners. No plane wave continuity constraints are enforced across the subdomain edges; this choice of the coarse level primal space does not satisfy Assumption 4. In our second test, in addition to the subdomain corner variables, we also include one edge average degree of freedom for each subdomain edge, as required in Assumption 4, in the coarse level primal variable space. This edge average degree of freedom corresponds to the vector determined by the cosine plane wave with the angle $\theta$ chosen perpendicular to the edge. In our last test, we further add to the coarse level primal space another plane wave continuity constraint on each edge corresponding to the cosine plane wave with the angle $\theta$ chosen tangential to the edge. In the following tables, we represent these three different choices of coarse level primal space by 0-pwa, 1-pwa, and 2-pwa, respectively.

Tables 3 and 4 show the GMRES iteration counts, corresponding to different number of subdomains, different subdomain problem sizes, and the three different choices of the coarse level primal space. With only subdomain corner variables in the coarse level primal space, the convergence cannot be achieved within 300 iterations in most cases. With the inclusion of the edge plane wave augmentations in the coarse level primal space, we see from Table 3 that the iteration counts decrease with an increase of the number of subdomains for a fixed subdomain problem size. We see from Table 4 that when the number of subdomains is fixed and $H / h$ increases, the iteration counts increase slowly, seemingly in a logarithmic pattern of $H / h$. Tables 3 and 4 also show that the convergence becomes slower with the increase of the shift $\sigma^{2}$ and that the convergence rate is improved by including more plane wave continuity constraints in the coarse level primal subspace.

\section{References}

[1] Yves Achdou and Frédéric Nataf. A Robin-Robin preconditioner for an advection-diffusion problem. C. R. Acad. Sci. Paris, 325, Série I:12111216, 1997.

[2] Xiao-Chuan Cai. Additive Schwarz algorithms for parabolic convectiondiffusion equations. Numer. Math., 60(1):41-61, 1991.

[3] Xiao-Chuan Cai and Olof Widlund. Domain decomposition algorithms for indefinite elliptic problems. SIAM J. Sci. Statist. Comput., 13(1):243258, January 1992.

[4] Xiao-Chuan Cai and Olof Widlund. Multiplicative Schwarz algorithms for some nonsymmetric and indefinite problems. SIAM J. Numer. Anal., 30(4):936-952, August 1993. 
Table 3. Iteration counts for symmetric, indefinite problems with $H / h=8$ and changing number of subdomains.

\begin{tabular}{|ccccc|}
\hline \multirow{2}{*}{$\sigma^{2}$} & & \multicolumn{3}{c|}{ Iteration Count } \\
\cline { 3 - 5 } 100 & \# subdomains & 0 -pwa & 1-pwa & 2 -pwa \\
\hline \multirow{3}{*}{200} & $16 \times 16$ & 183 & 37 & 14 \\
& $24 \times 24$ & 205 & 20 & 7 \\
& $32 \times 32$ & $>300$ & 13 & 6 \\
\hline \multirow{3}{*}{400} & $16 \times 16$ & $>300$ & 143 & 112 \\
& $24 \times 24$ & $>300$ & 85 & 39 \\
& $32 \times 32$ & $>300$ & 47 & 28 \\
\hline \multirow{3}{*}{$2 \times 16$} & $>300$ & $>300$ & 236 \\
& $24 \times 24$ & $>300$ & $>300$ & 75 \\
& $32 \times 32$ & $>300$ & 192 & 49 \\
\hline
\end{tabular}

Table 4. Iteration counts for symmetric, indefinite problems with $24 \times 24$ subdomains and changing $H / h$.

\begin{tabular}{|ccccc|}
\hline \multirow{2}{*}{$\sigma^{2}$} & & \multicolumn{3}{c|}{ Iteration Count } \\
\cline { 3 - 5 } 100 & $H / h$ & 0-pwa & 1-pwa & 2-pwa \\
\hline \multirow{4}{*}{200} & 12 & 205 & 20 & 7 \\
& 16 & 182 & 27 & 8 \\
& 8 & $>300$ & 85 & 39 \\
\hline \multirow{4}{*}{400} & 12 & $>300$ & 108 & 60 \\
& 16 & $>300$ & 114 & 68 \\
& 12 & $>300$ & $>300$ & 75 \\
& 16 & $>300$ & $>300$ & 108 \\
& & $>300$ & $>300$ & 111 \\
\hline
\end{tabular}

[5] Jean-Michel Cros. A preconditioner for the Schur complement domain decomposition method. In Domain decomposition methods in science and engineering, Proceedings of the 14th International Conference on Domain Decomposition Methods, pages 373-380. National Autonomous University of Mexico, 2003.

[6] Clark R. Dohrmann. A preconditioner for substructuring based on constrained energy minimization. SIAM J. Sci. Comput., 25(1):246-258, 2003.

[7] Clark R. Dohrmann. A substructuring preconditioner for nearly incompressible elasticity problems. Technical Report SAND2004-5393, Sandia National Laboratories, Albuquerque, New Mexico, October 2004. 
[8] Stanley C. Eisenstat, Howard C. Elman, and Martin H. Schultz. Variational iterative methods for nonsymmetric systems of linear equations. SIAM J. Numer. Anal., 20 (2):345-357, 1983.

[9] Yannis Fragakis and Manolis Papadrakakis. The mosaic of high performance domain decomposition methods for structural mechanics: Formulation, interrelation and numerical efficiency of primal and dual methods. Comput. Methods Appl. Mech. Engrg, 192(35-36):3799-3830, 2003.

[10] Thomas J.R. Hughes, Leopoldo P. Franca, and Gregory M. Hulbert. A new finite element formulation for computational fluid dynamics. VIII. The Galerkin/least-squares method for advective-diffusive equations. Comput. Methods Appl. Mech. Engrg., 73(2):173-189, 1989.

[11] Jing Li and Xuemin Tu. Convergence analysis of a balancing domain decomposition method for solving interior Helmholtz equations. Technical Report LBNL-62618, Lawrence Berkeley National Laboratory, May 2007.

[12] Jing Li and Olof B. Widlund. BDDC algorithms for incompressible Stokes equations. SIAM J. Numer. Anal., 44(6):2432-2455, 2006.

[13] Jing Li and Olof B. Widlund. FETI-DP, BDDC, and block Cholesky methods. Internat. J. Numer. Methods Engrg., 66:250-271, 2006.

[14] Marcus Sarkis and Daniel B. Szyld. Optimal left and right additive Schwarz preconditioning for minimal residual methods with Euclidean and energy norms. Comput. Methods Appl. Mech. Engrg., 196:1507-1514, 2007.

[15] Andrea Toselli. FETI domain decomposition methods for scalar advection-diffusion problems. Comput. Methods Appl. Mech. Engrg., 190(43-44):5759-5776, 2001.

[16] Andrea Toselli and Olof B. Widlund. Domain Decomposition Methods Algorithms and Theory, volume 34 of Springer Series in Computational Mathematics. Springer Verlag, Berlin-Heidelberg-New York, 2005.

[17] Xuemin Tu. A BDDC algorithm for a mixed formulation of flows in porous media. Electron. Trans. Numer. Anal., 20:164-179, 2005.

[18] Xuemin Tu. A BDDC algorithm for flow in porous media with a hybrid finite element discretization. Electron. Trans. Numer. Anal., 26:146-160, 2007.

[19] Xuemin Tu and Jing Li. A balancing domain decomposition method by constraints for advection-diffusion problems. Commun. Appl. Math. Comput. Sci., 3:25-60, 2008. 